\title{
Pre-analytical, analytical, and post-analytical considerations while processing samples of COVID-19 patients: Perspective from a clinical chemistry laboratory in India
}

\author{
Saswati Das', Bidhan Chandra Koner ${ }^{2}$ \\ Department of Biochemistry, ${ }^{1}$ Atal Bihari Vajpayee Institute of Medical Sciences and Dr. Ram Manohar Lohia Hospital, \\ ${ }^{2}$ Maulana Azad Medical College and Lok Nayak Jai Prakash Hospital, New Delhi - 110 001, India
}

Background: Since its detection in December 2019 in the city of Wuhan, China, the COVID-19 pandemic has grappled the world. Laboratories have been central in the diagnosis, prognosis and therapeutic monitoring of COVID-19 patients. With this pandemic spreading, laboratory personnel have become vital in the efforts to halt the advance of the virus. Aims and Objective: In this article, we will address various concerns in all the three phases of testing of the COVID-19 blood and serum samples in a clinical chemistry laboratory and discuss the issues pertaining to a resource-limited setting. Materials and Methods: International, national and regional guidelines on bio-safety and operational management available at the time of preparation of this manuscript were analyzed. Additionally, literature search through PubMed was done on the keywords "biosafety", "pre-analytical", "analytical", "post-analytical" and "COVID-19". Results: The pre-analytical, analytical, post analytical, general management problems we faced in our laboratory and the best practices that we followed to address the same has been included in this paper. Conclusion: Some unprecedented challenges the laboratories have encountered during this outbreak are maintaining the lab safety of highest standards, management of human resource while sustaining the quality of testing. Laboratories will have to constantly innovate in order to meet the demands of this outbreak.

Key words: Pre-analytical; Analytical; Post-analytical; Bio-safety; COVID-19

\section{Access this article online}

\section{Website:}

http://nepjol.info/index.php/AJMS DOI: 10.3126/ajms.v11i5.29296

E-ISSN: 2091-0576

P-ISSN: 2467-9100

Copyright (c) 2020 Asian Journal of Medical Sciences

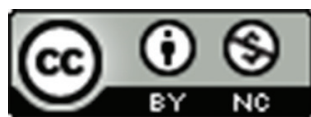

This work is licensed under a Creative Commons Attribution-NonCommercial 4.0 International License.

\section{INTRODUCTION}

In the month of December 2019 a zoonotic coronavirus crossed species to infect human populations. ${ }^{1}$ The pathogen has been identified as a novel enveloped RNA beta-coronavirus that has currently been named severe acute respiratory syndrome coronavirus2(SARS-CoV-2). ${ }^{2}$ According to the recent statistics of the World Health Organization (WHO), the disease also known as COVID-19, has spread across continents, with $6,416,828$ cases, and 382867 deaths as on June 4, 2020. ${ }^{3}$ Laboratory diagnosis plays an important role in the diagnosis and management of COVID-19. Real-time reverse-transcription polymerase chain reaction (rRT-PCR) is a nucleic acid amplification (NAAT) test that ensures direct virus identification whereas rapidimmune-detection tests (RDT) enables serological surveillance. ${ }^{4}$

The role of laboratory diagnostics extends beyond etiological diagnosis and epidemiologic surveillance, whereby biochemical monitoring through in vitro diagnostic testing are commonly used for assessing disease severity, for defining the prognosis, for guiding treatment andfor therapeutic monitoring. ${ }^{5}$ Common biochemical tests like Lactate Dehydrogenase(LDH), Alanine Aminotransferase(ALT), Aspartate Aminotransferase(AST), Procalcitonin(PCT), 
Bilirubin, Creatinine, Cardiac Troponin, D-Dimer, Ferritin, Prothrombin time, C-Reactive Protein(CRP), Interleukin-6 (IL-6)are being monitored in the suspected/positive COVID-19 cases. ${ }^{6,7}$ During infectious disease outbreaks, especially when they assume the relevance of pandemic disease such as COVID-19, the consequence of diagnostic errors are unwittingly amplified. ${ }^{8}$ Hence, the aim of this article is to provide an overview on the pre-analytical, analytical and post analytical challenges encountered while processing samples of patient with COVID-19 infection in a clinical chemistry laboratory, in a resource limited setting and provide the possible cost effective solutions.

\section{General laboratory considerations}

An area, activity and human resource specific risk assessment is essential by laboratories before formulating the bio-safety guidelines, in order to alleviate the risks during this pandemic.A separate laboratory area should be designated for processing of COVID-19 samples wherever possible. This laboratory area should have an accession zone and be equipped with centrifuge, auto-analyzers and other required instruments required for set up.In view of managing the increasing number of samples we have restructured our emergency/stat laboratory into a designated COVID-19 lab.Manpower management is one of the major challenges during the pandemic, as increased workload is anticipated. Hence, laboratory teams need to be separated with shift duties, which would also decrease crowding in the work benches. Staggering of meal timings and social distancing is encouraged. ${ }^{9} \mathrm{~A}$ contingency plan is prepared in accordance with CLSI GP36-A guideline, envisioning the scenario of staff members being sick or the team being quarantined. ${ }^{10} \mathrm{~A}$ standby team should always be ready in the event of a team being quarantined. According to WHO guidelines all specimens (swabs, body fluids, blood, and faeces) are to be considered potentially infectious, and appropriate PPE must be donned while obtaining samples from the suspected patients or processing such samples. ${ }^{11}$ Typically, PPE includes a long sleeved disposable gown, protective eye-gear, face shield, N95 respirator mask, gloves, cap, shoe covers. Hand washing is recommended before donning of PPE and after removal. Designated PPE donning and doffing areashould be demarcated in the vicinity of the laboratory having posters demonstrating the correct donning and doffing technique. This area should be equipped with designated waste disposal bins/bags. PPE is to be discarded as per the Biomedical Waste (BMW) Policy of the hospital. Staff training and competency assessment should continue during this period using e-resources. Biosafety training and training on processing the COVID-19 samples to be conducted at regular intervals in order to keep the laboratory personnel up-to-date. Technical team meetings and management review meeting may be conducted by video conferencing or other e-mode to ensure smooth functioning of the laboratory.

\section{Pre-analytical concerns}

Sample should be collected in an appropriate container which should be properly labelled. Sample volume should be enough for the advised laboratory tests and collected undertaking standard precautions to prevent hemolysis. The World Health Organization(WHO) guidelines recommend a 'triple packaging system' to contain an infectious material, which consists of a primary container typically a tightly sealed vaccutainer, a second leak-proof packaging and a third outer layer of packaging used to prevent physical damage to the secondary packaging while in transit. ${ }^{12}$ In our setting the second packing is usually a zip lock pouch and the tertiary packing is a thermocol box or specimen transportation box. After the third layer of packaging the specimen has to be labelled with a biohazard sticker and handed over to a trained manual carrier. On receiving the sample in the lab, the specimen transport box is sprayed with $0.1 \%$ sodium hypochlorite and left as it is for 10 minutes. The pneumatic tube system, if available should not be used for sample transportation, decreasing the chance of spillage. Electronic test requisition is preferable wherever possible if Hospital Information system (HIS) is in place, if not then test requisition form should be scanned and mailed to the laboratory via a secure e-mail service. The laboratory should set up an alert for the mails sent ensuring the priority of processing the samples. Accessioning of the samples to be done by trained laboratory staff member in PPE.Preventive maintenance of lab equipment may be done more frequently to avoid break down in this critical period.

\section{Analytical concerns}

PPE must be worn at all times while working in the laboratory. Potentially infectious materials should be handled by trained personnel only.All technical procedures (e.g. centrifugation, de-capping, sample dilution, secondary aliquoting) should be performed in a way that decreases the formation of aerosols and droplets. All suspected or COVID-19 positive patient samples are to be processed in full PPE, within a class II biological safety cabinet (BSC). Routine diagnostic testing of specimens can be handled in a Biosafety Level 2 laboratory.This is recommended in accordance with the Centers for Disease Control and Prevention as well as the WHO guidelines. ${ }^{13}$ Centrifugation ifrequired should be done in a sealed centrifuge. An air purifying respirator may be installed in the centrifugation area. In the unavailability of automated de-capper in a track system, de-capping of vaccutainers to be done in a class II BSC. Since de-capping is an aerosol generating procedure a face shield is recommended during the procedure in the event of non availability of BSC. The 
auto-analyzer designated for processing the COVID-19 samples should preferably have a wide menu to prevent secondary aliquoting.Routine calibration checks and quality control protocols are to be followed before starting the testing procedure. After processing the suspected samples, wash cycle of the auto-analyzer is to be run and daily maintenance procedures completed. A log of the same has to be maintained. A back up analyzer should be available and ready to be used in case of any analyzer downtime. Following analysis and processing, it is recommended that all surfaces, work benches and equipment are cleaned with an appropriate disinfectant. The WHO recommended disinfectants with proven activity against enveloped viruses are sodium hypochlorite $0.1 \%$, a minimum of $62 \%-71 \%$ ethanol, $0.5 \%$ hydrogen peroxide ammonium or phenolic compounds. ${ }^{14}$ All spills, accidents and potential exposures to infectious material must be reported to the laboratory in-charge immediately and a record of any such event is to be maintained. Spill management to be done with $1 \%$ hypochlorite solution $(10,000 \mathrm{ppm})$ for minimum 30 minutes and thenwiped clean. All work benches and equipment must be decontaminated after specimens have been processed with $0.1 \%$ sodium hypochlorite (1000 ppm) for general surface disinfection. All infectious material to be disposed in designated bins/bags labeled with biohazard sticker which will prioritize the removal of the waste from the laboratory surroundings. Analyzer waste to be pre-treated and disposed as per the BMW policy of the hospital. The BMW policy takes into account the guidelines set by central pollution control board, Govt. of India guidelines for the disposal of COVID-19 generated waste, ${ }^{15}$ a discussion on the same is beyond the scope of this paper.

\section{Post-analytical concerns}

Reporting of the test results to be done preferably electronically using the hospital information system, when not available, using alternative e-mode. Critical results are to be informed to the clinician at once, telephonically and a critical call $\log$ is to be maintained. Adhering to the laboratoryturnaround time (TAT) for the tests is absolutely essential as most of the COVID-19 patients are critical. Any delay in the TAT is to be communicated to the concerned clinical team telephonically.Laboratory stewardship protocols to be initiated in the hospital and rational use of laboratory tests absolutely necessary for clinical management and following the minimum retesting interval norms is advocated.

\section{Laboratory store management}

Because of many critically ill patients, total number of routine tests may increase to some extent despite decrease in samples from other patients due to lockdown. However, few tests like ferritin, PCT, CRP, IL-6, cardiac troponins etc. are expected to be requisitioned more in number.
So checking the trend of use of such tests and keeping adequate stock of these reagents are crucial. Because of lockdown, disruption of supply chain specially imported chemicals may affect stock position in ware house and local delivery by the suppliers may be delayed. But panic buying and hoarding may affect services in other labs treating such patients. Checking stock of PPE, masks and respirators, Sanitizers, disinfectants etc are also important in view of their increased use. Hence, judicious maintenance of stock position and keeping in touch with suppliers and alternative suppliers to know about stock position in their warehouse is advised to provide a smooth laboratory care service.

\section{CONCLUSION}

The laboratory management of COVID-19 samples is a work in progress, as there are still many concerns which remain unaddressed. We look forward to experiences from other regions on handing this crisis. It is needless to say quality of the total testing process (TTP) remains the top priority in the midst of the pandemic. Protecting the laboratorians is also a matter of grave concern during the pandemic. Hence, sticking to the bio-safety guidelines is of outmost importance. Repeated training of the laboratory staff on the bio-safetyguidelines may help mitigate the conundrum of maintaining a safe working environment. More tests may be added in future to predict, diagnose and monitor the prognosis of COVID-19; the laboratory should be constantly evolving and ready to tackle such challenges in future.

\section{REFERENCES}

1. Pneumonia of unknown cause - China: disease outbreak news. Geneva: World Health Organization, January 5, 2020 (https:// www.who.int/csr/don/05-january-2020-pneumonia-of -unkowncause-china/en/).

2. Guan WJ, Ni ZY, Hu Y, Wen-hua Liang, Chun-quan Ou, Jianxing $\mathrm{He}$, et al. Clinical Characteristics of Coronavirus Disease 2019 in China. N Engl J Med 2020.

https://www.nejm.org/doi/full/10.1056/NEJMoa2002032

3. World Health Organization. Novel Coronavirus (2019-nCoV) situation reports.https://www.who.int/emergencies/diseases/ novel-coronavirus-2019/situation-reports[Accessed: 4 Jun 2020].

4. Lippi G and Plebani M. The novel coronavirus (2019-nCoV) outbreak: think the unthinkable and be prepared to face the challenge. Diagnosis (Berl) 2020. Jan 28. https://doi.org/10.1515/dx-2020-0015

5. Lippi G, Plebani M. A modern and pragmatic definition of laboratory medicine. Clin Chem Lab Med 2020 Feb 18. https://doi.org/10.1515/cclm-2020-0114

6. Lippi $G$ and Plebani M. Laboratory abnormalities in patients with COVID-2019 infection. ClinChem Lab Med. Mar2020. pii: /j/ cclm.ahead-of-print/cclm-2020-0198/cclm-2020-0198.xml https://doi.org10.1515/cclm-2020-0198 
7. Rodriguez-Morales AJ, Cardona-Ospina JA, GutiérrezOcampo E, Villamizar-Peña R, Holguin-Rivera Y, EscaleraAntezana JP, et al. Clinical, laboratory and imaging features of COVID-19: A systematic review and meta-analysis. Travel Medicine and Infectious Disease. 2020 Mar 13:101623.

8. Lippi G, Simundic AM and Plebani M. Potential preanalytical and analytical vulnerabilities in the laboratory diagnosis of coronavirus disease 2019 (COVID-19). Clin Chem Lab Med. https://doi.org/10.1515/cclm-2020-0285

9. Tan SS, Yan B, Saw S, Lee CK, Chong AT, Jureen R, et al. Practical laboratory considerations amidst the COVID-19 outbreak: early experience from Singapore. J Clin Pathol March 2020 doi:10.1136/jclinpath-2020-206563

10. Planning for Laboratory Operations During a Disaster; Approved Guideline. CLSI document. GP36-A. Wayne, PA: Clinical Laboratory Standards Institute 2014

11. World Health Organization. Laboratory testing for 2019 novel coronavirus (2019- nCoV) in suspected human cases. Available: https://www.who.int/publications-detail/laboratorytesting-for-2019-novel-coronavirus-in-suspected-human-cases-
20200117[Accessed 4 Jun 2020]

12. World Health Organization. Guidance on regulations for the transport of infectious substances 2019-2020. Geneva: World Health Organization, 2019(WHO/WHE/ CPI/2019.20).

13. Centers for Disease Control and Prevention. Interim laboratory biosafety guidelines for handling and processing specimens associated with coronavirus disease 2019 (COVID-19). Available: https://www.cdc.gov/coronavirus/2019-ncov/lab/labbiosafety-guidelines.html[Accessed 4 Jun2020].

14. World Health Organization. Laboratory biosafety guidance related to the novel coronavirus (2019-nCoV), 2020. Available: https://www.who.int/publications-detail/laboratory-biosafetyguidance-related-to-coronavirus-disease-2019-(covid-19) [Accessed 4 Jun 2020]

15. Guidelines for handling, treatment and disposal of waste generated during treatment/diagnosis/quarantine of COVIID19 patients. Central pollution control board. Ministry of environment, forest \& climate change.Government of India.Available: https:// www.tnpcb.gov.in/pdf_2020/BMW-GUIDELINES-COVID_ Revisied_April2020.pdf[Äccessed 4 Jun 2020]

\section{Authors Contribution:}

SD- Concept, design, drafting the manuscript, critical revision; BCK- Concept, design, drafting the manuscript, critical revision.

\section{Work attributed to:}

Dr Ram Manohar Lohia Hospital, Maulana Azad Medical College.

Orcid ID:

Dr. Saswati Das- (1) https://orcid.org/0000-0002-4548-0066

Source of support: None, Conflicts of Interest: None 